\title{
Multifractal Fits to the Observed Main Belt Asteroid Distribution
}

\author{
Adriano Campo Bagatin ${ }^{1,2}$ \\ Vicent J. Martínez ${ }^{3}$ \\ Silvestre Paredes ${ }^{4}$
}

1. Departamento de Física, Ingeniería de Sistemas y Teoría de la Señal,

E.P.S.A., Universidad de Alicante

Apartado de Correos 99, 03080 Alicante, Spain

2. Physikalisches Institut, Universitaet Bern

Sidlerstrasse 5, 3012 Bern, Switzerland

3. Observatori Astronòmic, Universitat de València

Av. Vicent Estellés s/n, Burjassot 46100, Valencia, Spain

4. Departamento de Matemàtica Aplicada y Estadística

Universidad Politécnica de Cartagena, Paseo Alfonso XIII, 30203 Cartagena (Murcia), Spain

Accepted for publication in Icarus 


\begin{abstract}
.
Dohnanyi's (1969) theory predicts that a collisional system such as the asteroidal population of the main belt should rapidly relax to a power-law stationary size distribution of the kind $N(m) \propto m^{-\alpha}$, with $\alpha$ very close to $11 / 6$, provided all the collisional response parameters are independent on size. The actual asteroid belt distribution at observable sizes, instead, does not exhibit such a simple fractal size distribution.

We investigate in this work the possibility that the corresponding cumulative distribution may be instead fairly fitted by multifractal distributions. This multifractal behavior, in contrast with the Dohnany fractal distribution, is related to the release of his hypothesis of self-similarity.
\end{abstract}

Keywords Asteroids - Collisional Physics - Planetesimals 


\section{Introduction}

A collisional system may be defined as a population of bodies of different sizes, interacting with each other through occasional collisional events, whose probabilities per unit time are given functions of the size of the projectile and the target. As a consequence of an impact, both bodies are converted into a set of fragments whose size distribution depends on the relative size of the colliding bodies, their relative impact velocity, and a number of collisional response parameters determined by the composition, mineralogy, and possibly also their previous collisional history (Davis et al., 1992, 1993, Asphaug, 1999, Durda and Flynn, 1999). In planetary science, at least five examples of such collisional systems are often discussed: accreting planetesimals, planetary rings, the asteroid belt, the Trojan swarms systems and the Edgeworth-Kuiper Belt. To some extent, the case of the asteroid belt is the simplest one, since collisional velocities are so high that impacts mainly give raise to disruptive processes.

A useful mathematical model for the collisional evolution of the asteroid belt was introduced by Dohnanyi (1969) and independently by Hellyer (1970; for the sake of brevity, we shall refer hereinafter to Dohnanyi's work only). This model is based on one crucial assumption: all the collisional response parameters must be size-independent, implying that the transition from cratering to fragmentation outcomes occurs for a fixed projectile-to-target mass ratio, and no self-gravitational reaccumulation of fragments is taken into account.

The most important result of Dohnanyi's work is that, since collisional process gives raise to a cascade of fragments shifting mass toward smaller and smaller sizes, a simple power-law mass distribution is approached. Dohnanyi's theory states that the number of bodies $d N$ within the mass interval $(m, m+d m)$ is proportional to $m^{-11 / 6} d m$, or - in the diameter interval $(D, D+d D)$ - to $D^{-7 / 2} d D$, with the proportionality coefficients decreasing with time as the disruptive processes go on. The relaxation to this stationary mass distribution is fast: in the asteroid belt it occurs over a time span much shorter than the age of the solar system. Other authors (Williams and Wetherhill, 1994; Paolicchi, 1994; and Tanaka et al., 1996) confirmed these results and stated that the stationary exponent of the resulting power-law distribution is independent on the model of the collisional outcome of the fragmentation process, as long as the model remains self-similar. Moreover, they showed that the value of the exponent itself is determined only by the mass-dependence of the collisional rate (Tanaka et al., 1996). Again, Williams and Wetherill (1994) have shown that the $-11 / 6$ exponent changes very 
little, less than $10^{-4}$, when Dohnanyi's collisional physics assumptions are varied in a substantial way, but again, if the whole process remains self-similar. Let's stress that all along this paper we talk of self-similarity in a physical sense, that is referred to the physical characteristics of the bodies taking part to the collisional evolution.

On the other hand, almost complete and reliable observational data for asteroids of size larger than some $10 \mathrm{~km}$ have been collected in the past from direct optical and radar observations. From the analysis of these available data it is straightforward to see that in the asteroid belt distribution such a predicted fractal pattern does not show up at all. The observed size distribution-when represented on a double logarithmic plot_- "bends" in between the large mass end and the completeness size, instead of being represented by a straight line, typical of a fractal behavior. This observed pattern has sometimes been mimicked by using some different power-law distributions matched at some transition size.

As a matter of fact, the basic assumptions of Dohnanyi's theory are not fulfilled in real asteroid collisional systems. Collisional response parameters are not size-independent at all (at least at sizes greater than a few $\mathrm{km}$ ), as will be explained in Sec.3. This affects the resulting mass distribution in a substantial way, (Fujiwara et al., 1989; Davis et al., 1989, 1994; Campo Bagatin et al., 1994; Campo Bagatin, 1998).

\section{Multifractal distributions.}

It is possible to model a fragmentation process by means of a multifractal description. To illustrate how this can be done, we shall use an analytical multifractal mass distribution introduced in the analysis of turbulence by Benzi et al. (1984) (see also Menevau \& Sreenivasan (1987), and Tel (1988)). Let us consider the unit interval and subdivide it into $k$ equal pieces of size $1 / k$. Assign to each

piece a probability, or measure, $\left\{p_{i}\right\}_{i=1}^{k}$ such that $\sum_{i=1}^{k} p_{i}=1$. We continue this process by subdividing each segment of length $1 / k$ into $k$ new segments of length $1 / k^{2}$; now the probabilities $\left\{p_{i}\right\}_{i=1}^{k}$ are assigned to each segment following the same deterministic pattern of the first state distribution. The measure associated to each of the new intervals is just the product of this new assigned value by the $p_{i}$ value of its parent interval. The subdivision procedure is repeated again and again. After $L$ steps, we have $k^{L}$ intervals of length $1 / k^{L}$, each with a measure being the product of the last $p_{i}$ assigned to that interval by all its ancestors. The distribution of the measure becomes very inhomogeneous after a few steps of this construction. This kind of process is named "self-similar" in mathematics, as it 
is generated always in the same way, but this should not be confused with the physical definition of "self-similarity" given in Sec.1. The distribution itself is a distribution of mass (see Falconer 1990) and illustrates an inhomogeneousmultifractal - distribution on a compact non-fractal support (the unit interval).

We can see in Fig. 1 how the concentration of mass (represented by the height of the bars) varies widely from one region to another within the unit interval, for the case $k=3$. (Note that if one of the values of the probabilities is zero, for example $p_{2}=0$, and the construction is continued indefinitely, the support of the multifractal measure should be the triadic Cantor set, a fractal with dimension $D=\log 2 / \log 3$.

In general, a given set is said to show a multifractal behaviour, if the corresponding statistical analysis takes into account the number of its elements as well as their spatial distribution. In the case shown here the analysis is made only on the number of elements - and the resulting distribution is multinomial - but we shall rather keep the term because the analysis as a whole is indeed a multifractal one.

On the other hand, many different physical phenomena related to the natural processes of multiplicative cascades have been explained in terms of fractal and multifractal distributions (Falconer 1990, Meneveau and Sreenivasan, 1987, Martinez 1990, Borgani 1993, Chiu and Hwa 1991, Takagi 1994). We introduce here a multifractal technique for the analysis of the collisionally evolved population of main belt asteroids as a natural tool to treat this kind of physical systems.

\section{Stationary distributions and self-similarity.}

If we think about the stationary distribution for any collisional system in terms of fractal distributions, we see it can be characterized by its fractal dimension $d_{0}$, that is by the absolute value of the exponent of the distribution $N(\epsilon) \approx \epsilon^{-d_{0}}$, where $\epsilon=m / M, m$ is a generic mass, $M$ is the whole mass-range considered and $N(\epsilon)$ is the number of objects with mass $m$. The explicit relationship modelling such a distribution in a collisional system-as expressed in terms of the number of objects $d N$ in the mass interval $d m$-is of the kind $d N(m, m+d m) \propto m^{-\alpha} d m$, with $\alpha=11 / 6$ under Dohnanyi's hypotheses. Normalizing over $M$ we can identify $d_{0}$ with $\alpha$, that is the fractal exponent coincides with the Dohnanyi stationary exponent.

The only reliable data about the distribution of the number of objects at a given size in the main asteroid belt are the ones corresponding to the size distribution of asteroids in the observable range, that is from a few $\mathrm{km}$-size to 
Ceres' $913 \mathrm{~km}$. The distribution of masses $(M)$ can be related to that of sizes (diameters, $D$ ) via the obvious relationship $M=\frac{\pi \rho D^{3}}{6}$, assuming a constant density $\rho$-likely between 1.5 and $3.5 \mathrm{~g} / \mathrm{cm}^{3}$ —and spherical shapes.

By analyzing this distribution, it can be easily checked that the observed size distribution is not at all a simple power-law (fractal) distribution (see Fig. 2). The fact that asteroids in this size range do not follow the analytical distribution claimed by Dohnanyi and others is not surprising to us. In fact, one of the hypotheses that lead to the fractal stationary distribution is released here: there is no self-similarity in the collisional processes of asteroids of observable sizes due to two main reasons.

(a) Fragmentation experiments and models suggest a set of scaling-laws in order to accomplish for a realistic description of the resistance of materials to high velocity fragmentation. A typical physical magnitude that is useful in characterizing fragmentation phenomena is the impact strength, defined as the threshold energy density (energy per unit volume) necessary to fragment a target in a way that the greatest fragment produced has $50 \%$ the mass of the target. Scaling laws take into account both effects due to: (i) gravitational self-compression, that is an increase of the strength $(S)$ due to the pressure exercised by the upper material layers upon the deepest ones, increasing with the square of target size (Davis et al., 1985): $S=S_{0}+A(\rho D)^{2}$ ( $S_{0}$ is the impact strength measured in laboratory experiments, $A$ is a constant); $(i i)$ the so-called strain-rate effect, which is suggested by the idea that the specific energy necessary for a breakup should decrease as the size of the target increases (Farinella et al., 1982, Housen et al. 1991, Housen and Holsapple 1999): $S \propto S / D^{q}$ ( $q$ ranges from $1 / 4$ to $1 / 2$ ).

(b) The self-gravity of celestial bodies, on the other hand, varies as well at different sizes, and one of its effects is that-apart from the self-compression quoted above - it directly affects the escape velocity, especially in targets of size greater than at least a few hundred meters (the transition size is not univocally determined, as discussed in Holsapple, 1994; Love and Ahrens, 1996; Melosh and Ryan, 1997; Benz and Asphaug, 1999): then-for a fixed mass of projectile to mass of target ratio $\left(M_{p} / M_{t}\right)$ - the mass ratio of the escaping fragments divided by the mass of the target is not independent on $M_{t}$ itself. In other terms, the specific energy for disruption $\mathrm{Q}_{D}^{*}$ (that is the energy per unit mass necessary to disperse "to infinity" half of the mass of the target) is a function of $M_{t}$. This effect, by itself, is a clear example of violation of the self-similarity condition. This significantly modifies the characteristics of the multiplicative cascade, con- 
trarily to what happens in systems in which reaccumulation is non-existing or negligible. But reaccumulation is a common collisional outcome for asteroids, the relative amount of reaccumulated asteroids at observable sizes has been estimated to range between 0.5 and 1 (Campo Bagatin et al., 2001). Finally, a third potential source for non-self-similarity can be deduced from the experimental results reported by many authors (Davis and Ryan, 1994, Nakamura and Fujiwara, 1991, Giblin, 1998), indicating a shallow mass-velocity dependence in the velocity distribution of the ejected fragments of a catastrophic collision.

¿From what shortly described here, it is obvious that the quoted effects imply lack of self-similarity in the physics of fragmentation at different sizes. The resulting stationary distribution may depart from the well-behaved fractal predicted by the Dohnanyi theory and it may need to be represented by the structured multifractal distributions proposed in this work.

\section{Multifractal analysis.}

The goal of this research is to point out and show that multifractal analysis may be a natural and useful tool to match the observed stationary distributions of collisional systems when self-similarity assumptions in the characteristics of the collisional cascade are not expected to hold.

The multifractal analysis, and the corresponding fits to the observed populations of the main asteroid belt were obtained by means of the following simple procedure. First, we set a size interval ranging from around $30 \mathrm{~km}\left(D_{\min }\right)$ to about $400 \mathrm{~km}\left(D_{\max }\right)$ for asteroid sizes, and we consider the corresponding cumulative population of estimated masses in the asteroid belt $\left(M_{\min }, M_{\max }\right)$. We have chosen this range because it is the more reliable as far as completeness of data is concerned. There are only 3 asteroids larger than $400 \mathrm{~km}$, but - due to their size - they are not likely to have been the outcomes of the collisional evolution process since the time it reached the present stationary regime, some 4.5 Gyr ago. Quantitatively, it can be easily checked from previous works on the collisional evolution of the asteroid belt (Campo Bagatin, 1998) that shattering and dispersal of Ceres-size objects, the ones that could produce any single 400-500 $\mathrm{km}$ body present in the actual asteroid distribution, is expected approximately once every 4-5 Gys. Since this means that only one of the asteroids larger than $400 \mathrm{~km}$ could have been contributing to the collisional cascade, our assumption of neglecting the largest bodies in the collisional process seems reasonable, thence they have not been included into our statistical study.

Then we look for a multifractal distribution described by a set of multinomial 
probabilities $\left(p_{i}=p_{1}, p_{2}, \ldots, p_{k}\right)$, such that any given term of the kind $p_{1}^{r_{1}} p_{2}^{r_{2}} \cdots p_{k}^{r_{k}}$ corresponds to some mass in the actual distribution, in such a way that the cumulative multiplicity of any given term is equal to the cumulative number of bodies of the corresponding mass. The choice of comparing cumulative distributions rather than differential ones is due to avoid comparing artificial subdivisions of the asteroidal mass range in discrete arbitrary bins, with the multifractal mass distribution. Multinomial terms are transformed to masses by scaling their range to the corresponding given mass range $\left(M_{\min }, M_{\max }\right)$, chosen accordingly to the size range from about $30 \mathrm{~km}$ to about $400 \mathrm{~km}$, as explained above.

In order to derive the probabilities $p_{i}$ we therefore perform a systematic search for sets of probabilities matching the observed distribution, according to an automatic least-squared method, and when we find sets that fulfill the matching conditions, we finally transform back masses to diameters in order to get the plots in Fig.2, in which the observed size distribution and the obtained fits are shown.

At the end of the systematic search we found that for some sets of $k, L$, and $p_{i}(i=1, k)$ (shown in Table 1), the matching between the observed distribution and the multifractal ones is fairly good. We found sets of probabilities fitting a large number of multifractal terms and asteroid masses (sizes), corresponding to low dimensions $k$, that is to small number of parameters represented by the probabilities $p_{i}$. That happened for $k=2$ and $k=3$, for which the observed distribution could be best fitted with up to 12 multifractal terms, depending on the different values of the multiplicative level $\mathrm{L}$.

The distributions corresponding to the best matching sets can be seen in Fig. $2 \mathrm{~A}$ to $2 \mathrm{D}$. The deviations of any cumulative multiplicity of each multifractal term from its corresponding cumulative asteroid number is normally below $20 \%$ in any of the $k=2$ and $k=3$ cases.

A source of uncertainty in the translation from multifractal terms to masses to diameters of asteroids is the one on the value of the density, that we assume to be $2.5 \mathrm{~g} / \mathrm{cm}^{3}$. On the other hand, no error bars are shown on plots for the number of observed asteroid at any given size, but it should be pointed out that at least two possible sources for uncertainty in the observed asteroidal size distribution (and the related mass distribution) may affect the present analysis and the sets of probabilities:

i) the estimation of the sizes of asteroids, obtained from observed magnitudes and assumed surface albedos; 
ii) the actual cumulative number of asteroids, that should be anyway rather reliable for sizes larger than $50 \mathrm{~km}$, for fixed estimations of albedo and density.

\section{Results and conclusions.}

A set of reliable fits of the proposed statistical distributions to the observable data for the cumulative size distribution of main belt asteroids larger than $20 \mathrm{~km}$ is shown in Fig. 2A through 2D. We have found out a set of different multifractal distributions that may match fairly well the current cumulative distribution of the asteroid belt at observable sizes. This is interesting both on an operative and a physical ground. In fact, if the technique introduced here is used for other sets of collisionally evolved systems, it could provide a useful tool to match such distributions in a reliable way, with no need of guessing different power-law ranges matched somehow to approximately reproduce the data, as it has been done up to now: all we need is the mass distribution, or the size distribution with given assumptions about the shape and density of the bodies.

¿From a physical point of view, the fact that it is possible to fit the population of the main asteroid belt - or of any other nonself-similar collisonal systemby means of a multifractal distribution, introduces the fresh idea that physical inhomogeneities in fragmentation processes produce multifractal structures in this kind of systems. That also shows clearly that in these cases the Dohnanyi's hypotheses cannot be assumed and his conclusions cannot be generally applied.

But, what is the exact physical meaning of the found probabilities $p_{i} \mathrm{~s}$ ? It is not obvious how they may be related to collisional physics and/or to the conditions for the evolution. We suggest that such a correlation is embedded in the collisional cascade process described by multifractal distributions, as it happens to be for other multiplicative systems. We are investigating this major issue, in order to relate quantitatively the multifractal parameters to the physical characteristics of collisional evolution, but we prefer, at this stage, to limit the present interpretation to a phenomenological analysis, and to postpone the answer to this main question.

As far as the asteroid belt is concerned, we observe that at sizes smaller than a few tens of $\mathrm{km}$ - that is outside the range of the present work - extrapolations of recent data (Jedicke and Metcalfe, 1998) show a quasi-fractal behavior. This is not in contrast with what we have found in the present work. In fact, in the size range in which self-similarity does not hold, a depart from a simple fractal behavior due to nonself-similarity is expected and observed, leading to the multifractal distribution that we have introduced in this work. On the other 
hand, as soon as self-similarity begins to show up-like it seems to happen for smaller size bodies, for which self-gravitational effects have no main influence - a fractal behaviour becomes possible, as Dohnany and the other quoted authors have shown in the past. Campo Bagatin et al. (1994) and Campo Bagatin (1998) also showed that the release of one of the simplifying Dohnanyi's hypothesisthat is the absence of a lower cutoff in the mass distribution of asteroids-lead to a "wavy" pattern in the finally evolved distribution. Here we have shown a consequence of releasing another simplifying hypothesis, that of self-similarity. The two effects could in principle combine in shaping the actual mass distribution of asteroids in the main belt, making the whole picture more difficult to put into a unique simple scheme. 


\section{References}

Asphaug, E. 1999, Survival of the weakest, Nature, 402, 127-128.

Benz, W., E.Asphaug 1999, Catastrophic disruptions revisited, Icarus 142, 5-20.

Benzi, R., G. Paladin, G. Parisi, A. Vulpiani 1984, On the multifractal nature of fully developed turbulence and chaotic systems, J.Phys.A 17 3521-3531.

Borgani S., G.Maurante, A. Provenzale, R.Valdarnini 1993, Multifractal analysis of the Galaxy Distribution: Reliability of Results from Finite Data Sets, Physical Review E 47 3879-3888.

Campo Bagatin, A., P. Farinella, and J-M. Petit 1994, Fragment ejection velocities and the collisional evolution of asteroids, Planet. Space Sci., 42, 1099-1107. Campo Bagatin, A. 1998, Collisional evolution of asteroidal systems, Ph.D. Thesis Servicio de Publicaciones Universidad de Valencia, Valencia, Spain.

Campo Bagatin, A., J.-M. Petit, P. Farinella 2001, How many rubble piles are in the asteroid belt?, Icarus 149, 198-209.

Chiu C.B., R.C. Hwa 1991, Multifractal structure of multifractal production in branching models, Physical Review D 43 100-103.

Davis, D.R., C.R. Chapman, S.J. Weidenschilling, and R. Greenberg 1985, Collisinal history of asteroids: Evidence from Vesta and the Hirayama families, Icarus, 62, 30-53.

Davis, D.R., P. Farinella, P. Paolicchi, S.J. Weidenschilling, and R.P. Binzel 1989, Asteroid collisional history: Effects on sizes and spins, in Asteroids II, eds. R.P. Binzel, T. Gehrels and M.S. Matthews (Univ. of Arizona Press), pp. 805-826.

Davis, D.R., P.Farinella, P. Paolicchi, A. Campo Bagatin, A. Cellino, and V. Zappalà 1992, Collisional effect of tiny asteroids on their larger siblings, Bull. Amer. Astr. Soc. 24, 961.

Davis D.R., P.Farinella, P.Paolicchi, A. Campo Bagatin, A. Cellino. and V. Zappalà 1993, Deviations from the straight line: Bumps (and grinds) in the collisionally evolved size distribution of asteroids, Lunar Planet. Sci. Conf. XXIV, $377-378$.

Davis, D.R., E.V. Ryan, and P. Farinella 1994, Asteroid collisional evolution: Results from current scaling algorithms. Planet. Space Sci., 42, 599-610. 
Donhanyi J.W. 1969, Collisional model of asteroids and their debris, J. Geophys. Res. 74, 2531-2554.

Durda, D.D., G.J.Flynn 1999, Experimental study of the impact disruption of a porous, inhomogeneous target, Icarus, 142, 46-55.

Falconer, K.J. 1990. Fractal geometry. Mathematical foundations and applications, John Wiley \& Sons Eds..

Farinella, P., P. Paolicchi, V. Zappalà 1982, The asteroids as outcomes of catastrophic collisions, Icarus, 52, 409-433.

Fujiwara, A., P. Cerroni, D.R. Davis, E. Ryan, M. Di Martino, K. Holsapple, and K. Housen 1989, Experiments and scaling laws on catastrophic collisions, in Asteroids II, Eds. R.P. Binzel, T. Gehrels and M.S. Matthews (Univ. of Arizona Press), pp. 240-265.

Giblin, I. 1998, New data on the velocity-mass relation in catastrophic disruption, Planet. Space Sci. 46, 356-364.

Hellyer, B. 1970, The fragmentation of the asteroids, Mon. Not. R. astr. Soc. 148, 383-390.

Holsapple, K.A. 1994, Catastrophic disruptions and cratering of the Solar System: A review and new results, Planet. Space Sci., 42, 1067-1078.

Housen, K.R., Holsapple, K.A. 1999, Scale effects in strength dominated collisions of rocky asteroids, Icarus, 142, 21-33.

Housen, K.R., R.M.Schmidt, and K.A.Holsapple 1991. Laboratory simulations of large scale fragmentation events, Icarus, 94, 180-190.

Jedicke R., and T.S. Metcalfe 1998, The orbital and absolute magnitude distribution of main belt asteroids, Icarus 131, 145-260.

Love, S.G., and T.J. Ahrens 1996, Catastrophic impacts on gravity dominated asteroids, Icarus, 124, 141-155.

Martínez V.J. 1990, Fractals and multifractals in the description of the cosmic structure, Vistas in Astronomy, 33, 337-356.

Melosh H.J., and E.V.Ryan 1997, Asteroids: Shattered not dispersed. Icarus, 129, $562-564$.

Meneveau, C. and K.R. Sreenivasan 1987, Simple multifractal cascade model for 
fully developed turbulence, Phys.Rev.Lett., 1424-1427.

Nakamura, A., Fujiwara, A. 1991, Velocity distribution of fragments formed in a simulated collisional disruption, Icarus, 92, 132-146.

Paolicchi, P. 1994, Rushing to equilibrium: A simple model for the collisional evolution of asteroids, Planet. Space Sci., 42, 1093-109\%.

Takagi, F. 1994, Multifractal structure of multiplicity distributions in particle collisions at high energies, Physical Review Letters 72 32-35.

Tanaka, H., S. Inaba, and K. Nakazawa 1996, Steady-state size distribution for the self-similar collision cascade, Icarus, 123, 450-455.

Tel, T. 1988, Fractals, multifractals and thermodynamics, Z. Naturforsch., 42a, 1154-1174.

Williams, D.R., and G.W. Wetherill 1994, Size distribution of collisionally evolved asteroid populations: Analytical solution for self-similar collision cascades, Icarus 107, 117-128. 


\begin{tabular}{|c|c|c|c|c|}
\hline Case & $\mathrm{A}$ & $\mathrm{B}$ & $\mathrm{C}$ & $\mathrm{D}$ \\
\hline $\mathrm{k}$ & 2 & 2 & 3 & 3 \\
\hline $\mathrm{L}$ & 11 & 12 & 9 & 11 \\
\hline$p_{1}$ & 0.818 & 0.833 & 0.737 & 0.789 \\
\hline$p_{2}$ & 0.182 & 0.167 & 0.168 & 0.178 \\
\hline$p_{3}$ & - & - & 0.095 & 0.033 \\
\hline
\end{tabular}

Table I The 4 sets of $k, L, p_{1}, p_{2}, p_{3}$ leading respectively to the fits represented in Fig. 4A, B, C, D.

\section{Figure captions}

Figure 1. A few steps of the construction of the self-similar multifractal measure described in the text by means of a multiplicative cascade. The values of the initial probabilities are $p_{1}=0.3534, p_{2}=0.4363$ and $p_{3}=0.2103$. The support of the measure is the unit interval. At each stage of the construction, the measure associated to each interval is represented by the height of the rectangular box. We show steps 1, 2, 4, and 8, from top to bottom and left to right. After several steps, the distribution of the measure becomes rather inhomogeneous, a characteristic pattern of multifractal distributions.

Figure 2. The four best fits to the main asteroid belt cumulative population of asteroids, using the multifractal technique explained in the text. The dashed lines show the observed distribution and the crosses represent the multifractal fits.

Fig. 2A to $2 \mathrm{D}$ correspond respectively to cases A to D shown in Table 1. 

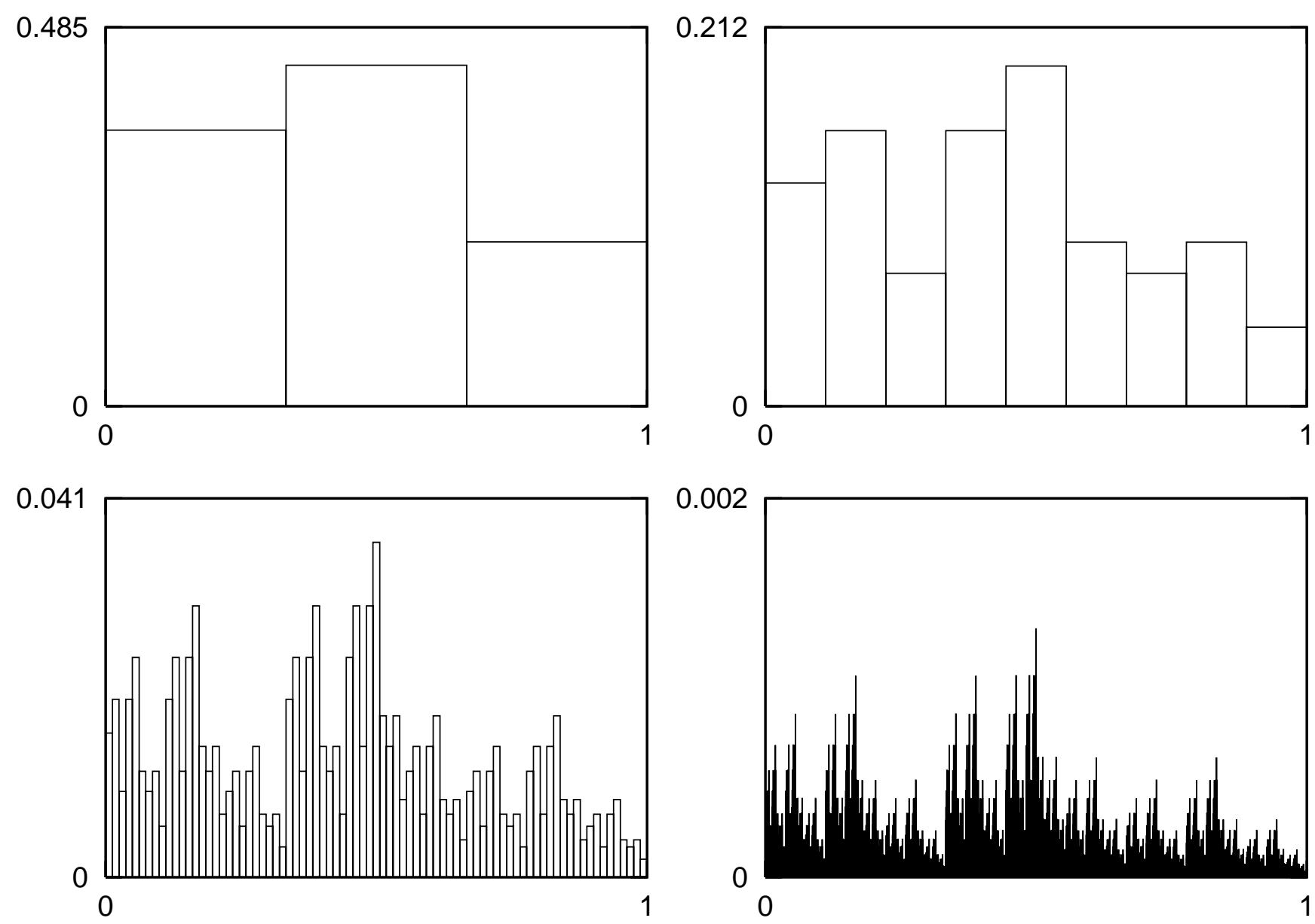

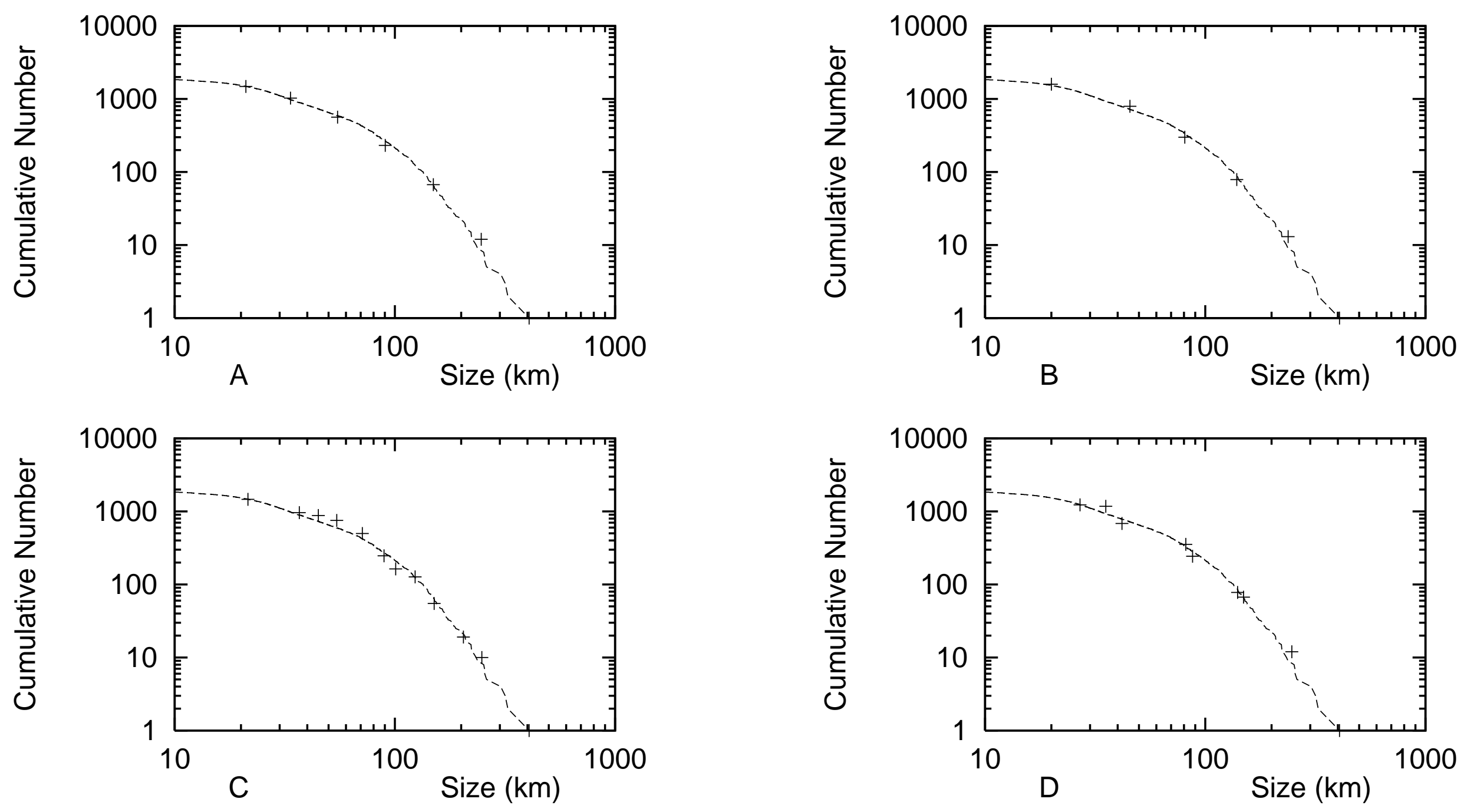